\title{
Aisthesis
}

Firenze University Press www.fupress.com/aisthesis

OPEN ACCESS

Citation: L. Sakse (2021) Distance: Closeness/Remoteness in Kim Boske's Photographs. Aisthesis 14(1): 71-80. doi: 10.36253/Aisthesis-12771

Copyright: (c) 2021 L. Sakse. This is an open access, peer-reviewed article published by Firenze University Press (http://www.fupress.com/aisthesis) and distributed under the terms of the Creative Commons Attribution License, which permits unrestricted use, distribution, and reproduction in any medium, provided the original author and source are credited.

Data Availability Statement: All relevant data are within the paper and its Supporting Information files.

Competing Interests: The authors have declared that no competing interests exist.

\section{Distance: Closeness/Remoteness in Kim Boske's Photographs}

\author{
LigA SAKsE \\ Latvian Academy of Culture, Riga, Latvia \\ ligasakse@gmail.com
}

\begin{abstract}
The work Moving Flowers \#1 by the internationally known Dutch artist Kim Boske (born in 1978) was published in the first cover series HOU VOL ("Keep Faith") of the periodical Het Parool on 30 May 2020. Following the aim of the newspaper to help cope with the disastrous consequences of COVID-19, the artist focused on how our perceptions had changed during the pandemic. If previously the content (or what is being represented) was the element that dominated in photography, then during the pandemic, the representational harmony of the content was replaced by the structural features or the way how something is being represented. The article aims to reflect on the artworks created by Boske in 2020 and to show the circulation of ideas contained in them, and with this case study to highlight more general trends in the recent art photography.
\end{abstract}

Keywords: aesthetics, photography, Kim Boske, perception of an image, COVID-19.

\section{INTRODUCTION}

The typical theme of Dutch painting - a still life of flowers - has attracted Boske's attention. The still life of flowers is an abundant theme that fascinates with the inexhaustible diversity. It is considered that the oldest known images of still life in the territory of Europe are the frescoes painted in Roman culture in around 62-69 BC. Though ancient frescoes are located in the territory of Italy, the genre of still life became popular mostly in the $17^{\text {th }}$ century Dutch and Flemish paintings. At that time, it was common to paint still lifes with flowers, game, generous tables with food and vanitas still lifes, which illustrated the transitory nature of the secular life. The attention of contemporary Dutch artists and photographers has been attracted by experiments with stage ${ }^{1}$ still life and conceptual interpretations, creating radiographs, hypercollages and conceptual

${ }^{1}$ Since the 1970s there has been a tradition in the Dutch photography to create staged still life photography. 
photography, as, for example, can be evidenced in the works of Arie van Riet, Bas Meeuws, Hans Withoos, Iris Gonzalez, Jeroen Luijt, Marie Cécile Thijs, Marnix Goossens, Mathilde Karrer, Ron van Dongen, Titus Brein, Yvonne Lacet and others. Kim Boske works among these artists, studying how physical movement in time and space constantly shifts our perspective to the physical world. By combining different perspectives into one image, the artist creates a multi-layered reality by maintaining an innovative approach and experimental aesthetics. To the question about her sources of inspiration, Boske replies:

I read the work of philosophers, such as Gilles Deleuze and Bergson. But I am also inspired by someone like Andrew Wiles, the mathematician who solved the thesis of Fermat. These people help to adjust and sharpen my perspective on the world. They keep my process going and make me notice and be inspired by different things every time, especially within nature (Boske [2014])

Boske's approach is to provide the audience with a sense of immersion and interaction of the image with the audience, since the main role in her photographic work is to capture time and space in a transformational way, by summing up several perspectives from a distance.

\section{REMOTENESS. THE TEMPORAL DIMENSION}

When reading Boske's book Mapping (Boske [2012]: 53-56), we find evidence that the artist has continuously developed individual technical approach by practically moving the camera in time and space when engaging in the process of creating conceptual photography, that is to say, by dematerializing the visible reality and giving it artistic quality. Boske explains the working process: "Also to make clear how the physical movement through time and space constantly changes our perspective on the world» (Boske [2019]). Does this reveal the signs of iconicity and does it mean that the representation is arbitrary and not similar to what the representation stands for? For instance, in the 2015 work Bouquets from the Golden Age (Fig. 1), Boske takes photographs from several perspectives. Forms taken from different perspectives are combined into one image so that the full volume of an object is discovered on a deep black background. By mechanically fixing many shots in one image, Boske creates a transition from the visible reality to an attempt to reach such a level in photo aesthetics, where one can see various forms and shades over time.

By focusing on the aesthetic features, which are integral to the colour photograph Bouquets from the Golden Age, the apparent combinations of its shades and air vibrations are revealed characteristic to the aesthetics of painting by the old masters. Despite the use of modern technologies, the aesthetics of the image seems to be tied with the sense of historical saturation radiated by the works of the $17^{\text {th }}$ century Flemish old masters. The aesthetics of Boske's works of photographic origin reaches such a level of shades and tones that it becomes a reference to the painteresque Rembrandt's colour palette that has been developed in the figurative oil painting and reminds of the unique existence of the work of art defined as "aura" by Walter Benjamin: ${ }^{2}$

In even the most perfect reproduction, one thing is lacking: the here and now of the work of artits unique existence in a particular place. It is this unique existence-and nothing else-that bears the mark of the history to which the work has been subject (Benjamin [2002]: 103)

Benjamin brought forward the question of the changes introduced by the technological development of the photographic image both in terms of the perception and diversification of the aesthetic experience. Boske is rather interested in the aspects of perception in terms of representation:

My work can be characterised as research into the system of time and space. I am fascinated by the sys-

\footnotetext{
2 Written late December 1935 beginning of February 1936; unpublished in this form in Benjamin's lifetime.
} 


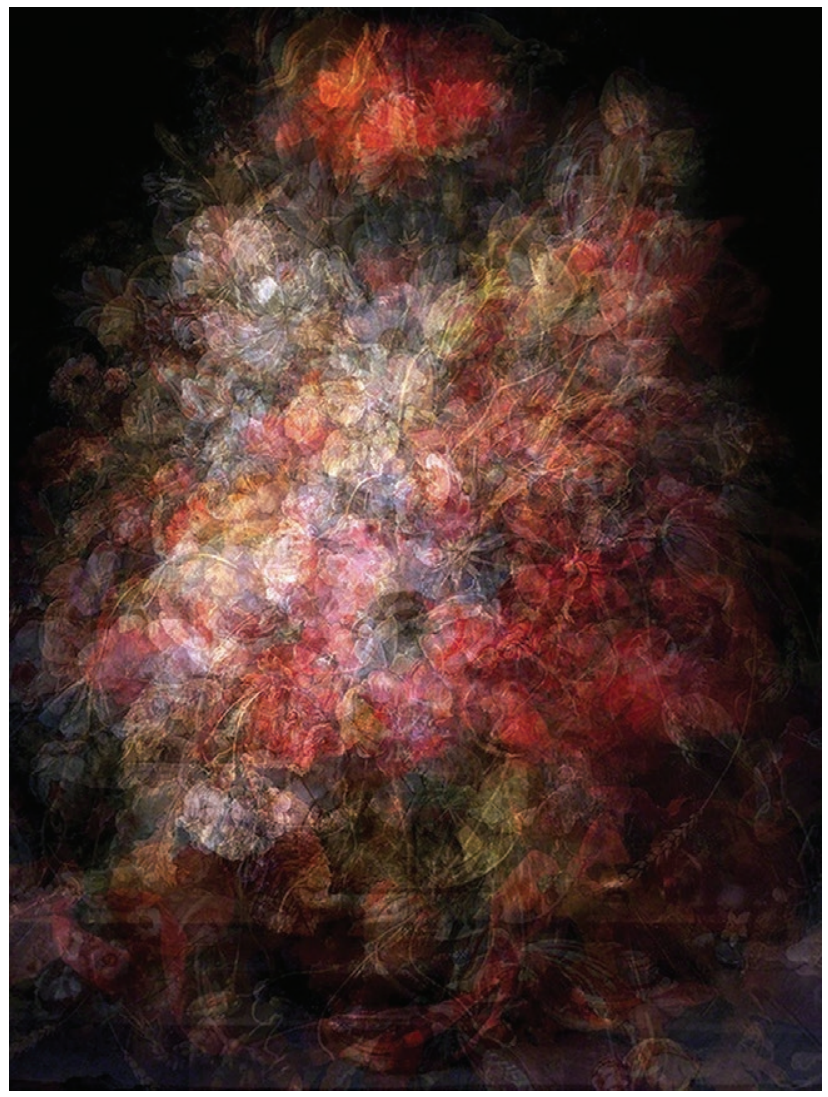

Fig. 1. Kim Boske, Bouquets from the Golden Age, (2015) Colour photography, $28 \times 21 \mathrm{~cm}$.

tems that exist behind the visible surface of the visual world (Boske [2019])

It should be acknowledged that her works have such an integrity of representation and at the same time the depth of the image that encourage the viewer to ponder. Her photographic image shows a combination of new features that considerably differ from the material, visual or aesthetic features of the initial object. Yet, it is not the same thinking model that is based in the Western visual culture and that is modelling clearly visible objects in real time. Consequently, a question emerges: if it is not an evident phenomenon, does the photographic object exist at all? However, the image exists in the perception of the viewer, who connects the essence of these objects with the meaning that they represent and not with the real form. Besides, the photographer points to the importance of the creative process claiming: «What fascinates me is a reality and a way of thinking that presents itself more as 'becoming' rather than "being"» (Boske [2014]).

\section{USE OF THE STRUCTURE OF NATURE. CLOSENESS}

On the one hand, Boske's photographs are based on the motifs of nature (a tree, a bush, flowers, a waterfall), yet, on the other hand, the photographs are not connected with a representation of one particular natural landscape, because the artist uses nature as a phenomenon that gives rise to the process of change and movement. She explains:

'Nature' has acquired a dominant position in my photographs as nature has a special relationship with chaos and order. Patterns in nature are never totally alike and they seem to never reappear in exactly the same way. Nature is overwhelming. There is a treasure of information, structures and processes hidden in nature (Boske [2014]).

In order to examine the works of 2020 , it is important to mention the fact that in 2018 Boske used an opportunity to work in Kamiyama, ${ }^{3}$ Tokushima, Japan, where she became familiar with Japanese culture. Taking into account that in Japan culture and nature are inextricably linked, Boske refers to such a component of culture as the Šintoismu or šintō ${ }^{4}$ (in Japanese かんながらのみ ち - kannagara-no-michi or 神道 - しんとう) tradition $^{5}$, which provides such an understanding of

${ }^{3}$ Kamiyama started its Kamiyama Artist in Residence (KAIR) program in 1999. Every autumn, three artists are sponsored to come and stay in the town to create art and interact with the local people for approximately two months. See: https://www.in-kamiyama.jp/en/art/.

${ }^{4}$ Šintō, indigenous religious beliefs and practices of Japan. https://www.britannica.com/topic/Shinto

${ }^{5}$ Emeritus Professor of Shintō Studies, Kokugakuin University, Tokyo Naofusa Hirai states: Shinto has existed in Japan without any founder. Kokugakuin University Encyclopedia of Shinto http://eos.kokugakuin.ac.jp/modules/ 


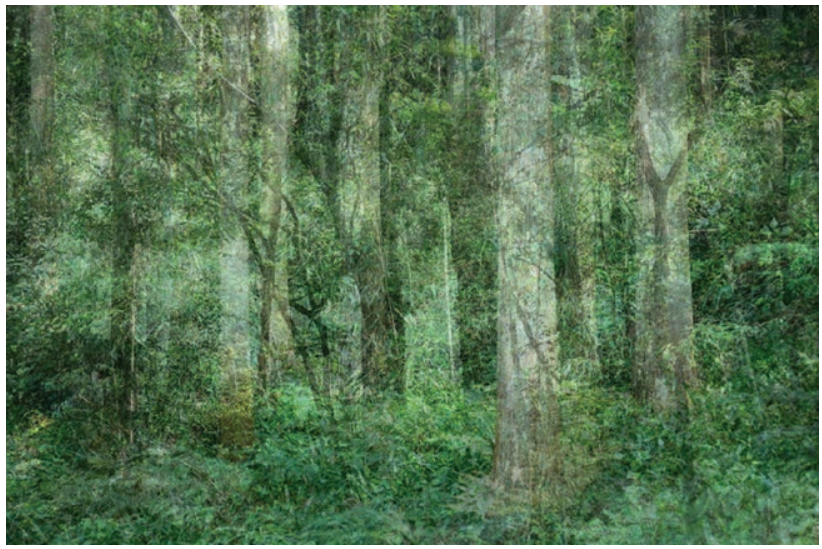

Fig. 2. Kim Boske, Shinrin-yoku 1 (2020), pigment print.

nature, where deification of nature and mythological beliefs form an integral part.

Since residency in Japan, Boske's works have been resonating with the Japanese culture: the natural indigo (Schueller 2021) farming, the workconsuming indigo fermenting process, the manual production of Japanese traditional paper, ${ }^{6}$ handmade washi paper, print dyed in natural indigo, at Awagami factory, Kamiyama, Tokushima, as well as the delicate working process, which entails the thinking pattern about the inheritance of traditions and the natural processes, thus literally integrating nature in her works. During the pandemic, she continues communicating with the colleagues of the residency, and cooperates in terms of the indigo dying traditions, which date back to the ancient world 1600 years BC.

Boske also ensures high-quality creative process in the framework of this tradition. Such global solidarity can be achieved along with the understanding that the cultural heritage must be preserved and the traditions must be cherished by understanding that the new expression has emerged on the basis of the long forgotten previous tradition. During the COVID-19 restrictions, Boske returned to the theme of trees in her work Shinrin-yoku 1 (Fig. 2) by revealing a scene of a

xwords/

${ }^{6}$ Washi (和 紙) is a traditional Japanese paper, which is made using a local fibre processed manually and made in a traditional way. forest, where the trees merge with each other and refer to the world, which is simultaneously the existing reality and a different reality in terms of the movement, tonal gradation and adventure revealing multiple meanings. By placing one layer of photography onto another that has been taken from a different angle, Boske finds her own conceptually autonomous image, which characterises the constant state of change in nature in the most vivid way.

In nature, organic objects are never entirely still. Each form of life is characterised by movement, for example, the movement of branches and leaves filters the light and provides both the light and shadow on the ground. Both the silhouettes of tree trunks and the plants close to the roots form the foreground. In addition, the branches and the green leaves fill the midsection and the background of the image, creating a dimension of depth, representing the total form as an atmospheric object. Taking into account the distance from the trees, Boske shows such a perspective that leaves both the tops and the roots of the trees at the layer of soil with the energy of life outside the frame. The frame includes the trunks of bigger and smaller trees as a metaphorical reference to shelter and nutrition provided by the oxygen-rich forest. The foliage rendered by Boske vibrates at the slightest deviation of light and is reproduced from detailed, smear-like shapes that encourage the viewer to look and calm down, providing balance and simultaneously maintaining the idea of an active healing power, or bathing in a forest. Forest Bathing (Shinrin-Yoku, jap. 森林浴) originated in Japan in the early 1980s, and may be regarded as a form of nature therapy and the positive effects of nature therapy are also indicated by the field studies in science (Park et al. [2010], Berger [2020]), emphasizing the crucial role of the tree as a collector of macro-particles and producer of oxygen.

In the work Shinrin-yoku 2 (Fig. 3) the abundant tonality is determined mostly by the presence of light onto the green leaves and stream, whose representation is rather abstract than objective, but it can be identified as the co-exist- 


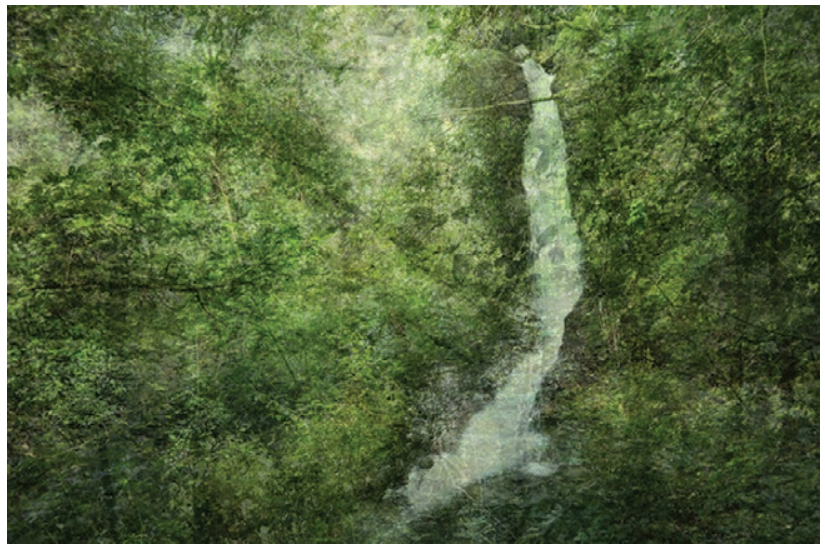

Fig. 3. Kim Boske, Shinrin-yoku 2 (2020), pigment print.

ence of the forest and mountain river as parts of the elements of the global natural order, witnesses of the past that are related with a harmonising archetype of Japanese natural landscape. When examining the artist's approach, Josephine van Schendel claims:

The so-called struggle of natural life, and survival of the fittest, should be interpreted in the sense of the ability to coexist and cooperate with complex relationships, rather than the ability to exploit and suppress. This serene thought of togetherness pops up in my mind when I hypnotizingly look at the shapeshifting landscape of Boske's layered photoworks. She seems to be a master of high-lighting the multiplicity of forms of life (van Schendel [2020]: 4).

If we talk about the artist's works as works of art, which shed the light on the essence, it is crucial to find out whether it could be the case that the restrictions caused by the pandemic have allegedly created unprecedented forms of practice in the aesthetics of Boske's works.

\section{INTERACTION BETWEEN TIME AND SPACE DURING THE PANDEMIC. CONTACT}

At the times, when due to the COVID-19 spread, national isolationism emerged, the restrictions determined by the countries also gave rise to the artists' needs to ensure creative process, at the same time keeping the opportunities of show- ing the work at the exhibitions. Boske in social media published photographs as an insight in the working process, adding such hashtags as \#kimboske, \#artcanhelp, \#coronavirus. In June 2020 she wrote:

What is currently happening brings me back to the essence of my work. [...] But it also means that we have more headspace. It includes boredom, too, but you can explain it with a heightened state of focusing and understanding. It inspires me. This heightened sense of awareness will taint our memories. If we are allowed to move on in full speed, I hope, part of this attention will remain. Speed, which we are used to in life, distances us from ourselves. Lingering takes us to the core and essence (van Zadelhoff [2020]:8-9).

The times of the pandemic are globally characterised as times of anxiety and adaptation to the environment with screens. Although it provides us with inexhaustible amount of information, it is also known that long-term screen consumption causes difficulties both to focus attention and to concentrate on the work that you have started. In these circumstances, the government carries out support measures. National museums are accessible online: "In April 2020, the Dutch government launched financial stimuli aimed specifically at supporting the cultural sector during the COVID-19 crisis [...]. These measures added to pre-existing generic measures aimed at protecting the employment of artists and professionals of the cultural sector during this time of profound crisis» (Boekman Foundation [2020]).

With the challenges arising from these circumstances, Boske provides an opportunity to focus on a specific case, helps to explore not only the temporal system, physical movements in time and space, but also how we perceive what we see and how it changes our view to the world. In the view of Boske, time is itself a structure consisting of differentiated structures, so in spring 2020 during the quarantine she implemented an idea to create a series of photographs Moving Flowers (Fig. 4, 5, 6, 7,8 ) of archival materials, which included the masterpieces of the $17^{\text {th }}$ century still life painting, freshly picked herbs and meadow flowers. As a pigment 


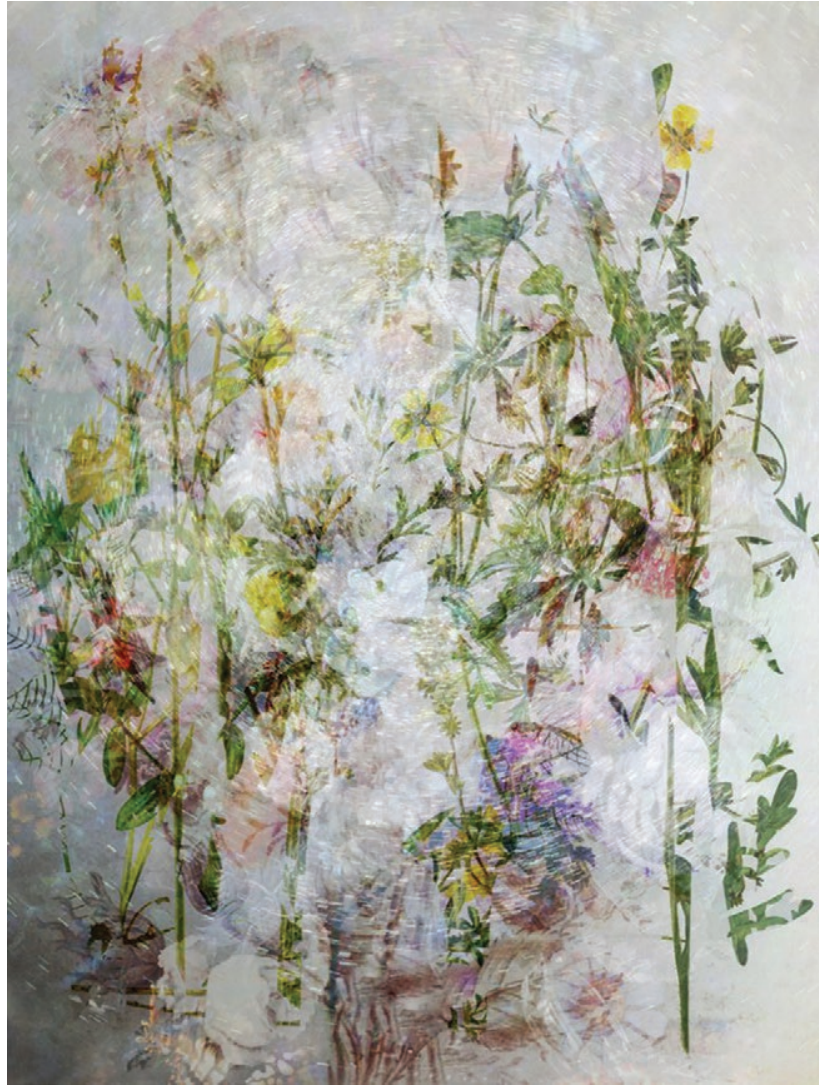

Fig. 4. Kim Boske, Moving Flowers \#1 (2020), photography Inkjet print in washi paper, edition of $7+2 \mathrm{AP}, 60 \times 45 \mathrm{~cm}$. Courtesy FLATLAND, Amsterdam.

print on the washi paper it was later processed with the natural indigo fermentation process.

In the finely elaborated time network Moving Flowers \#1 (Fig. 4), all images have been combined so that a new image would be created, as a result giving rise to associations with the represented phenomenon. Out of five works of the series, Moving Flowers \#1 is the lightest in terms of tonality and the most translucent. Perhaps, for this reason it was chosen for the first cover layout of the Dutch periodical Het Parool $^{7}$ on 30 May 2020 as a stimulus for the battle with the devastating consequences of the pandemic. The shapes of Boske's multi-layered photographic works intuitively reveal that natural phenomena have equal rights to exist, grow roots, bloom, wither and engage in the network of nature relations.

${ }^{7}$ Het Parool is an Amsterdam-based daily newspaper.

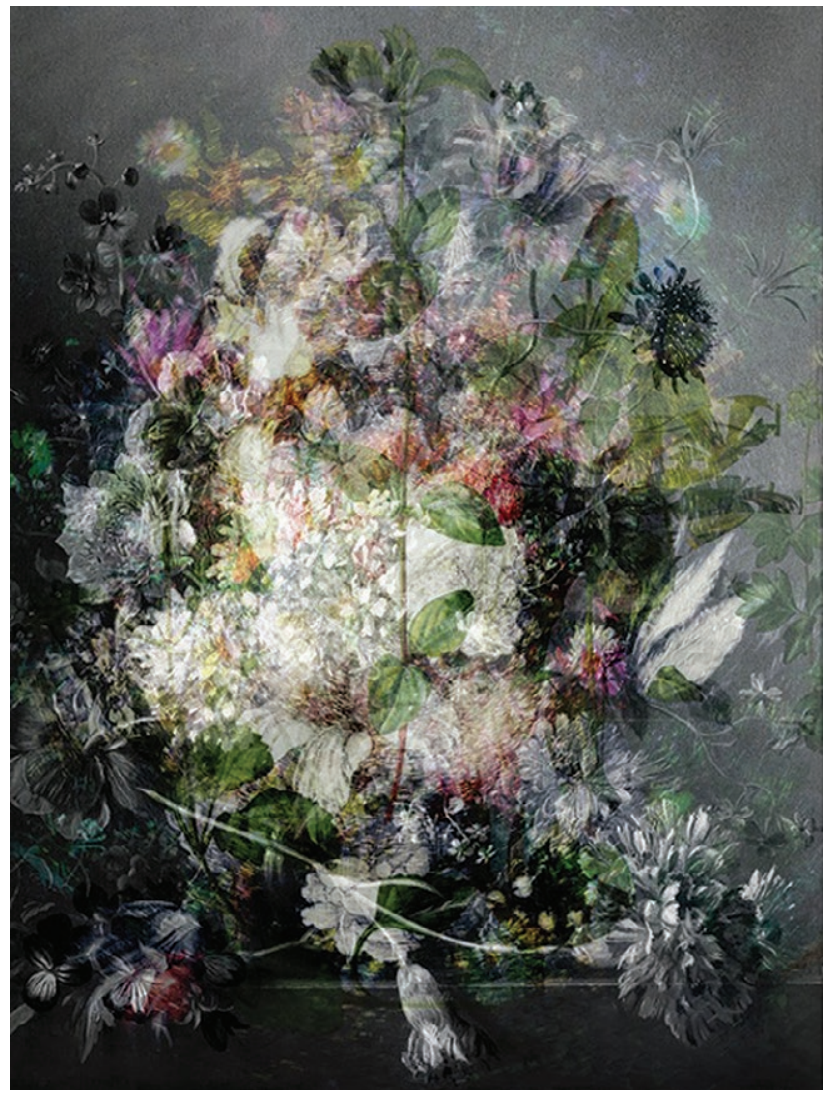

Fig. 5. Kim Boske, Moving Flowers \#2 (2020), photography Inkjet print in washi paper, edition of $7+2 \mathrm{AP}, 60 \mathrm{x} 45 \mathrm{~cm}$, Courtesy FLATLAND, Amsterdam.

Boske admits that every piece of her art is created in a time-consuming process in which she tries to achieve and to ensure that the viewer would stay longer by noticing the sense of time. The longer you look, the more and more you see the hidden, multi-layered and complicated world embedded in the image structure. She made the image Moving Flowers \#2 (Fig. 5) by analogy with Flemish masterpieces, using the principle in which flowers (carnations, hydrangeas, irises, anemones, tulips, meadow clovers and other summer meadow flowers) were arranged in a vase from plants available in different seasons, which in reality would never have been able to bloom at the same time. In her work Moving Flowers \#3 (Fig. 6) the object has at the same time many perspectives representing the photographed fern leaves, painted hollyhocks that overlap and are 


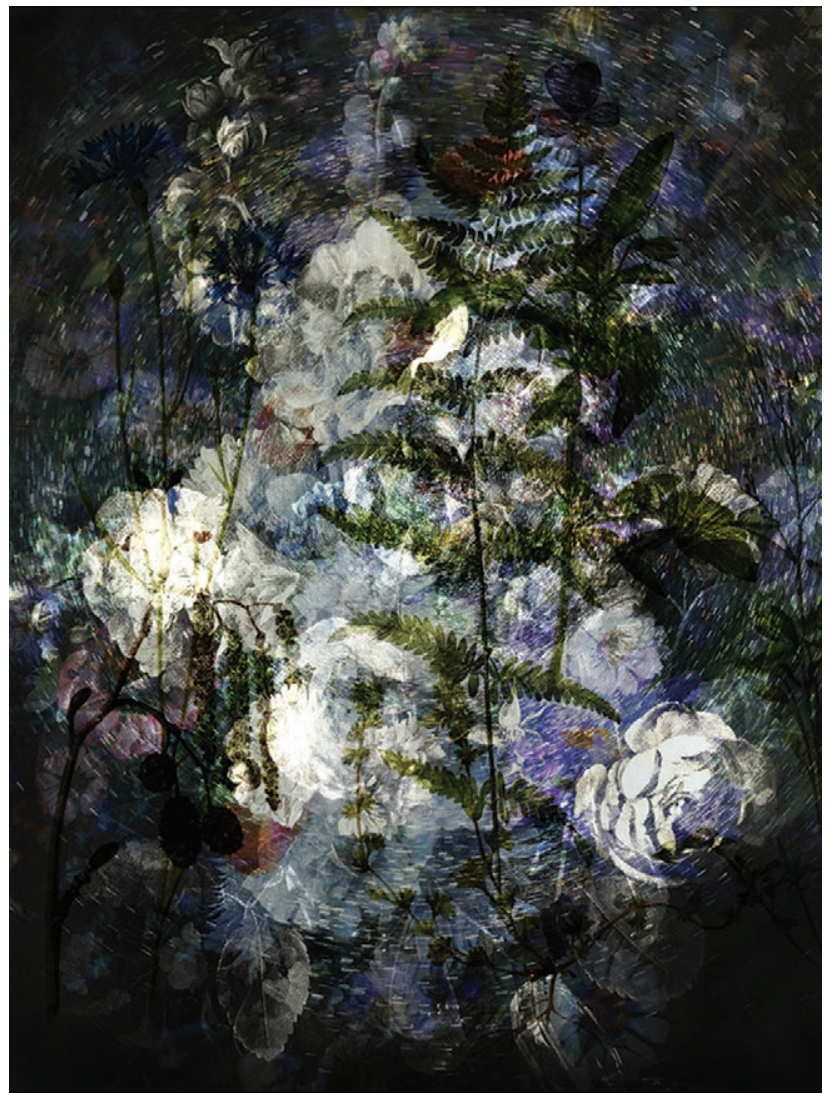

Fig. 6. Kim Boske, Moving Flowers \#3 (2020), photography Inkjet print in washi paper, edition of $7+2 \mathrm{AP}, 60 \times 45 \mathrm{~cm}$. Courtesy FLATLAND, Amsterdam.

merged in the image, as if simulating the movement of appearance and disappearance.

Technically analysing Boske's multi-layered images, each separate layer has a clear focus and a sharp image. Besides, the spectator's knowledge gives rise to associative links and develops awareness of details and presence.

Photographic image Moving Flowers \#4 (Fig. 7 ) is tonally the most colourful of the works in this series - it is seemingly a still image, yet it continuously changes due to the colour relations and work construction. Without affecting the overall unity of the image, along with the presence of the indigo pigment onto the washi paper, shapes and tones are revealed to the spectator gradually. Both the translucent texture of the flowers chosen by the author and the indigo colouring process with coded metaphorical refer-

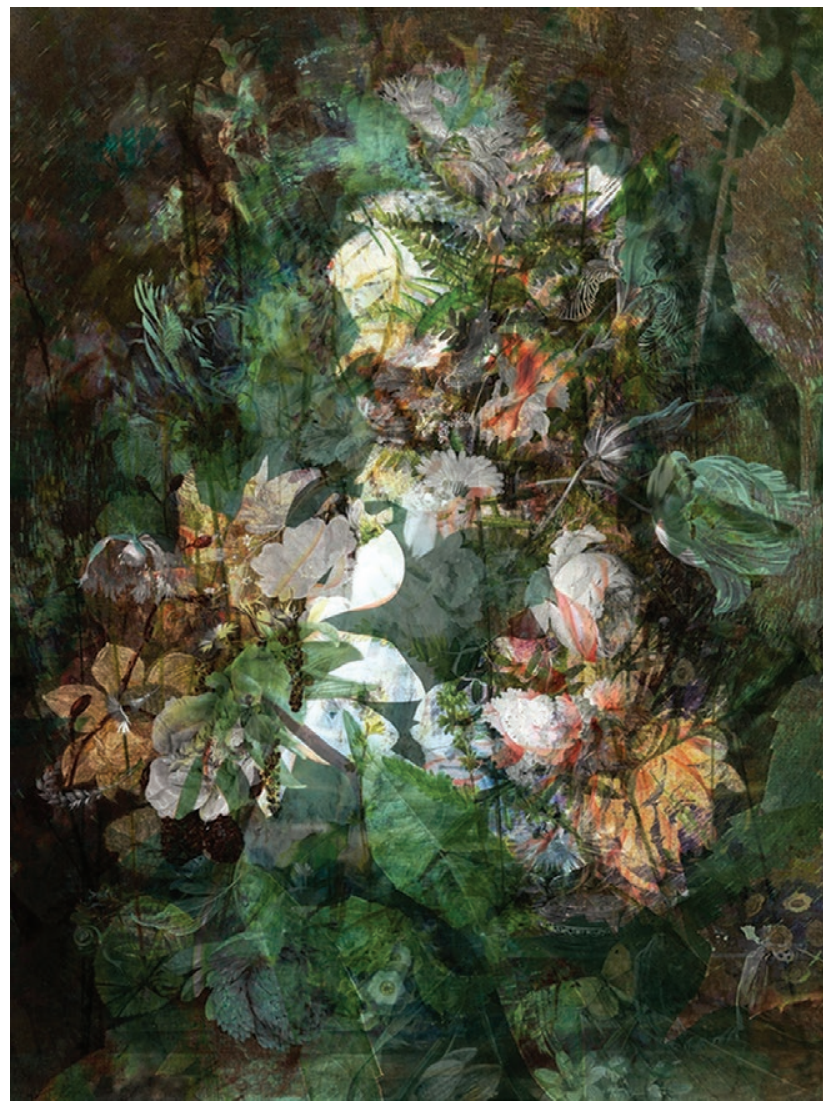

Fig. 7. Kim Boske, Moving Flowers \#4 (2020), photography Inkjet print in washi paper, edition of $7+2 \mathrm{AP}, 60 \mathrm{x} 45 \mathrm{~cm}$. Courtesy FLATLAND, Amsterdam.

ences resonate with the continuity of the natural cycles characterised by variability, movement and continuation.

The closing work of the series - Moving Flowers \#5 (Fig. 8), as it can be seen in the printout on the washi paper, is the most richly saturated in terms of tonality in the process of natural indigo colouring. In one of the drawing layers of the image, there are clearly marked circular lines with a dashed stripe resembling the images of long-exposed stars photographed in the night sky. This centred circular movement reveals that natural processes are connected with everything that exists and, although the relations between man and nature are complicated, it is a natural order, where the death is not the end in the framework of life processes, but the beginning of continuation. 


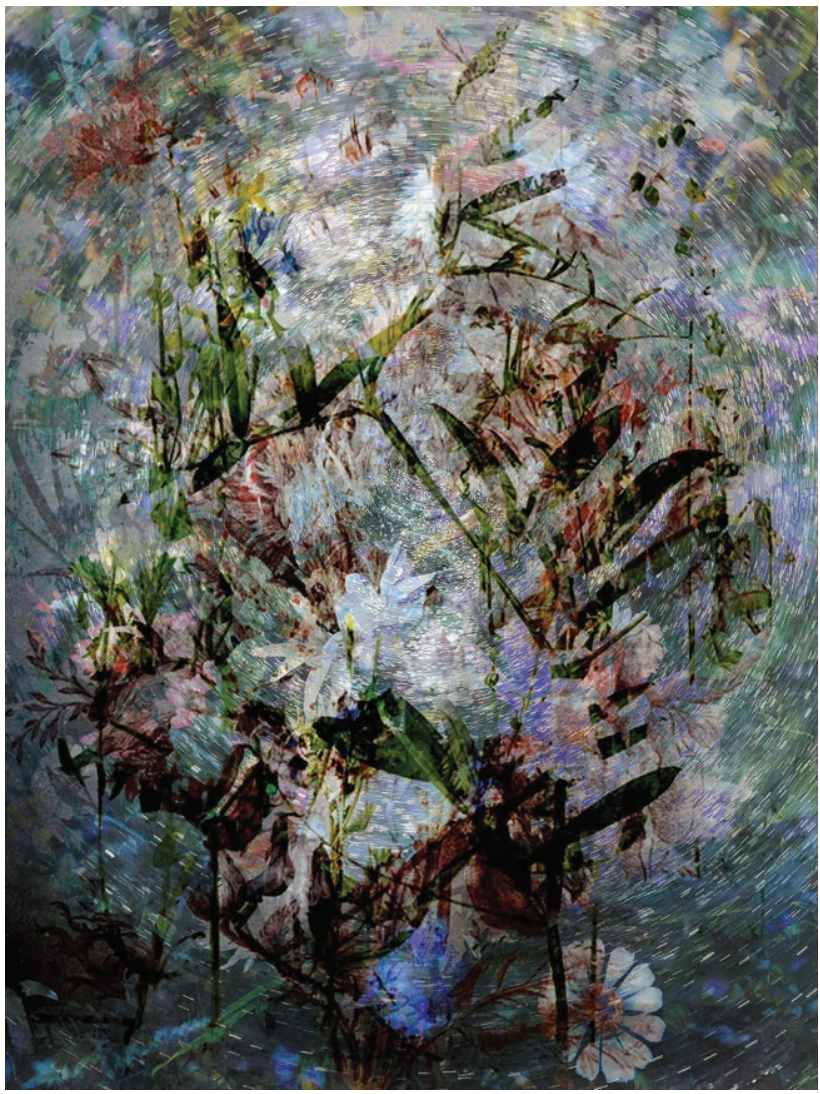

Fig. 8. Kim Boske, Moving Flowers \#5 (2020), photography Inkjet print in washi paper, edition of $7+2 \mathrm{AP}, 60 \times 45 \mathrm{~cm}$. Courtesy FLATLAND, Amsterdam

Finally, let's focus on Boske's work Akui Gawa \#1 (Fig. 9) created in the vertical format. In the work nearly three metres long the artist is exploring the movement of the river stream, which is a combination of many photographs revealing changes in the water flow and resembling the transcendent river flow as a state of continuity, essence of change and state of vitality. In the framework of the rich Eastern visual culture, Boske associatively reflects on the merged areas typical of ancient Chinese silk paintings, as well as the use of proportions, composition and vertical format known in the Japanese aesthetics. With the work Akui Gawa \#1, which has been printed out on the washi paper and has been painted by the artist herself in an indigo bath made in her studio, Boske participated in an exhibition in the gallery FLATLAND, which received audi-

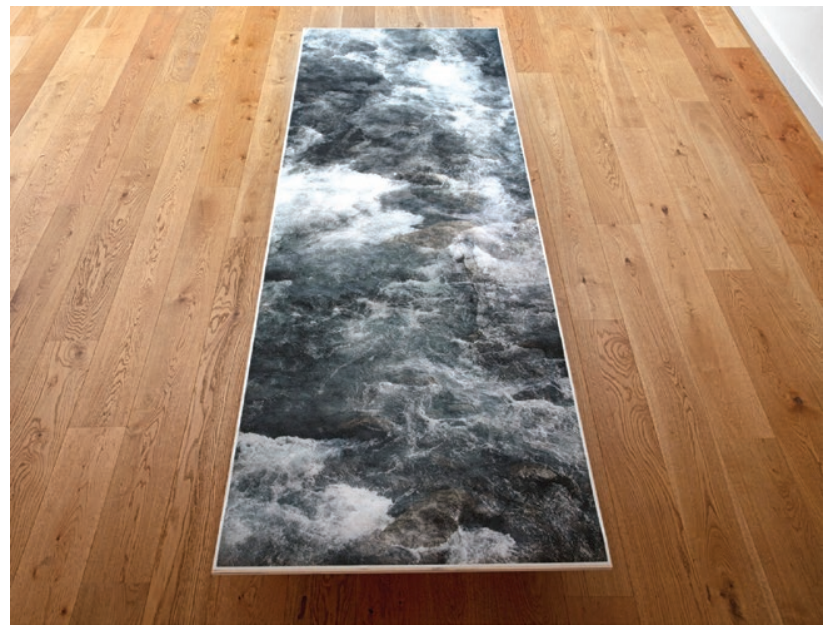

Fig. 9. Kim Boske, Akui Gawa \#1 in the exhibition Trancendence: Outside Time (2020), inkjet print on washi paper in artist frame, 282 x $94 \mathrm{~cm}$. Courtesy FLATLAND, Amsterdam.

ences according to the previously made appointment list. Artists Kim Boske and Valérie Belin participated in the exhibition with works about transcendence as an object of research. In 2021, the following idea was expressed about the exhibition Transcendence - Outside Time at the gallery FLATLAND: «Their work can be described as trans-semiotic: they use iconic signifiers to challenge the expectation of the viewer and let us rethink the way we inhabit the world». 8

\section{CONCLUSIONS}

It is obvious that the effects of the pandemic COVID-19 have resulted in the paradigm shifts at a global level - in political, economic, social and cultural aspects - but for the representatives of visual arts this time has been a real challenge, because the meaning of creative work has had to be seriously reconsidered.

For Boske, this means using innovative practices to continue to create by maintaining links and interaction with both unreachable colleagues in Japan and with the audience overall, taking into account the need to exhibit and incorporate works into arts affairs, both on-site and

${ }^{8}$ See http://www.kimboske.com/News. 
off-site models - in Art Rotterdam (February, 2020), POST Bookshop Tokyo (July, 2020), Art Paris (September, 2020), Photo London (October, 2020), Mondriaan Foundation (December, 2020) and FLATLAND (December, 2020). It should be acknowledged that, due to the limits of the pandemic, the participation in PHOTO Paris and the personal exhibition INDIGO at the Tokushoma Museum of Modern Art, scheduled for 2021, have been cancelled.

The creative process of Boske followed up in the context of the pandemic in 2020 and the solutions that the artist has undertaken in her studio in Amsterdam, in order to create photographs Shinrin-yoku 1 and Shinrin-yoku 2, make series Moving Flowers out of five works, as well as largescale printout on the washi paper Akui Gawa \#1, it should be recognised that during the global quarantine Boske is able to focus on new works, activates the digital potential and applies unprecedented practices in the aesthetics of her works. She continuously develops and reinforces the use of technical means (maintaining fermentation processes in the indigo bath, dyeing with natural indigo colour).

The case study of the series Moving Flowers additionally reveals that it would be a mistake to talk about the discourse of the Dutch still life in visual arts as old-fashioned. In general, in the era of the overload of screen aesthetics, which explicitly points to the convergence of visual arts and the advertising world, Boske shows an independent conceptual work. Besides, Moving Flowers proves that she uses the still life as one of the many available strategies.

The revolutionary approach of the artist towards to the temporal dimension attracts the interest of the field professionals, because Boske has not exhausted her potential and the still life of flowers exists both as a powerful archetype and recognisable and important example in the practice of conceptual photography. The aspects of attention conception contained in Boske's works that have been discovered in the context of the spread of pandemic, takes the audiences to the aesthetic experience based in time and space: distance, closeness/remoteness and contact. Consequently, both in the art photography and in its analysis, such categories as closeness/ remoteness and contact have become crucial. It is possible to discuss them under one umbrella term - distance.

The Dutch photographer offers a new artistic reality to the viewer, in which both spatial and temporal perspectives are synthesised in a single image, so that its structure does not copy at any given moment and place the objective reality, but constitutes a different surface structure of conceptual photography revealing the changes in spatial perception during the COVID-19 spread.

\section{REFERENCES:}

Benjamin, W., 1936: The Work of Art in the Age of Technological Reproducibility, in Id., Selected Writings: Volume 3 1935-1938, Cambridge, Belknap Press of Harvard University Press.

Berger, R., 2020: Nature Therapy: Incorporating Nature Into Arts Therapy, "Journal of Humanistic Psychology”, 60 (2), pp. 244-257.

Bergson, H., 1889: Time and Free Will: An Essay on the Immediate Data of Consciousness, transl. by F. L. Pogson, Harper and Row, New York, 1960.

Boekman Foundation, 2020: How will the cultural sector emerge from the corona crisis?Available:https://www.boekman.nl/en/ wpcontent/uploads/sites/2/2020/04/Summary-How-will-the-cultural-sector-emergefrom-the-coronacrisis-28-April.pdf (viewed 19.02.2021.)

Boske, K., 2014: An Interview with Kim Boske, “Madame Figaro" Japon (フィガロ ジャポン) 2014年 06月号 [囚誌]. Available: http://www. kimboske.com/Texts (viewed 20.01.2021.)

Boske, K., 2012: Mapping, Text - Essay On trees and Men, ed. by Dr. Erik A. de Jong, Mondriaan Foindation, Amsterdam.

Bury, S., 2019: The Artist's Book in the Age of Digital Reproduction: Walter Benjamin and the Artist's Book, "Arts", 8, 138. 10.3390/arts8040138. 
Boske, K., 2019: The Artist Statement, Available: http://www.flatlandgallery.com/artists/kimboske/biography/ (viewed 16.01.2021.)

Deleuze, G., 1983: Cinema 1: The MovementImage, transl. by $\mathrm{H}$. Tomlinson and B. Habberjam, Athlone Press/Minneapolis, University of Minnesota Press, London, 1986.

Dilven, A., Kruisbergen, L., 2019: Transcript from a short film about Kim Boske Nothing Really Ends, www.kimboske.com/Newwork. Available:https://vimeo.com/359229804 (viewed 11.06.2021.)

Hirai, N. (Invalid Date), Shintō. Encyclopedia Britannica. Available:https://www.britannica.com/ topic/Shinto (viewed 10.06.2021.)

Park, B.J., Tsunetsugu, Y., Kasetani, T., Kagawa, T., Miyazaki, Y., 2010: The physiological effects of Shinrin-yoku (taking in the forest atmosphere or forest bathing): Evidence from field experiments in 24 forests across Japan, "Environmental Health Preventive Medicine", 15, pp. 18-26.

Schueller, R., Indigo. How Products Are Made. Retrieved March 20, 2021 from Encyclopedia. com Available:https://www.encyclopedia.com/ manufacturing/news-wires-white-papers-andbooks/indigo (viewed 10.02.2021.)

Van Schendel, J., 2020: Deep Ecology and Boske's Clandestine Landscapes, "FLATLAND Journal", 8, December, Amsterdam. p. 4. Available:https://www.flatlandgallery.com/cms/ wp-content/uploads/2021/01/Flatland-Journalhigh-res.pdf (viewed 11.06.2021.)

Van Zadelhoff, R., 2020: An Interview with Kim Boske, "FLATLAND Journal", 7, June, Amsterdam. pp. 8-9. Available:https://www.flatlandgallery.com/cms/wp-content/uploads/2020/06/ FlatlandJournal_Seventh-edition-2020_bw.pdf (viewed 10.06.2021.) 